\title{
VIII. Health Diagnostics
}

Improvements in life style and progress in medicine have been responsible for a large increase of life expectancy during the last two centuries. Today, in developed countries, people live much longer than they did in previous eras. In France, for example, the life expectancy before the French revolution (1789) was less than 30 years. It reached $5^{\circ}$ years in 1900 and has consistently improved since that period. Today it is above 80 years. In underdeveloped countries, the life expectancy is lower and can be of the order of 40 years in the poorest countries or in those where large segments of the populations are faced with a diseases such as AIDS or alcoholism, for example. As populations become older deaths due to chronic diseases increase. About $80 \%$ of seniors have at least one chronic disease and $50 \%$ have at least two. In the United-States $70 \%$ of all deaths are due to chronic diseases. Technological advances such as those offered by nanotechnology can have far reaching consequences in healthcare.

The main issue in the health domain is to provide an affordable and efficient medicine that all can access. For our discussion we can classify health care can be classified into three main areas: diagnostics, therapeutics and restoration or regenerative medicine (figure 67).

Proper medical diagnoses usually require some combination of analysis and imaging. The current trend is to employ non-invasive or minimally invasive techniques to look inside the human body or to make specific analyses on samples of material taken from the patient. Diagnostics techniques should be as painless as possible so that they can be performed routinely and used to identify threats of serious disease. In the case where a sample is needed to perform an analysis, it is also desirable that the volume of the sample be as small as possible.

Once a disease or health disorder is identified, it is necessary to treat the patient in the most efficient way. In the therapeutics stage it is important to deliver the right quantity of drugs to the right place and at the right time in order to minimize side effects and increase treatment efficiency. Monitoring the efficiency of the treatment using minimally invasive diagnostic methods is also required.

Accidents or diseases can damage or destroy parts of the human body. Further, as people live longer and their bodies age, repair or replacement of components such as organs, bones or teeth may become necessary. The third domain of medicine, restorative and regenerative medicine, addresses such needs. 


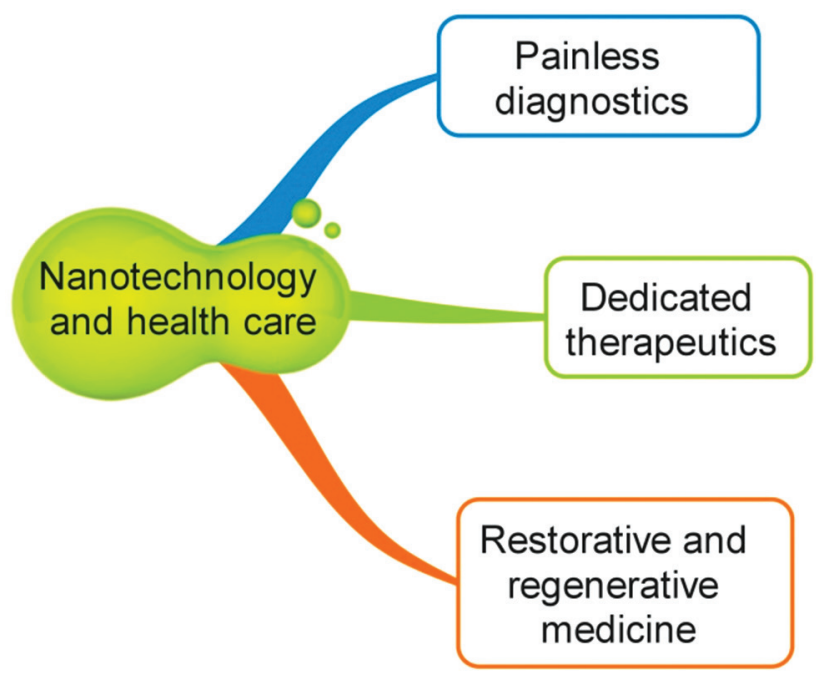

Figure 67. Nanotechnology can have impact in the three domains of medicine shown.

Nanotechnology can play a role in each of the health domains specified by providing improved diagnostics enabling early detection of or prevention of diseases and making more efficient techniques for treatment and follow-up monitoring available.

\section{Major diseases}

While there are many health risks and all must be addressed, it is possible to categorize the major diseases that affect very large proportions of the human population. All have a large social and economic impact. These are shown in figure 68.

The leading cause of death in developed regions of the earth such as the United States and The European Union is cardiovascular disease. Cancer ranks as the second leading cause of death. Neurodegenerative diseases such as Alzheimer's or Parkinson's are a serious problem. Due to dietary and lifestyle factors Type II diabetes is an increasing health problem. Inflammatory and degenerative joint diseases as well as musculoskeletal disorders lower the quality of life of many people. These diseases demand a long-term medication. Bacterial infections require new strategies to complement antibiotics because some bacteria are becoming resistant to them. For economic reasons, little research is now devoted to antibiotics. Fighting against viral infections also requires novel treatments. Both bacterial and viral infections require quick detection. 


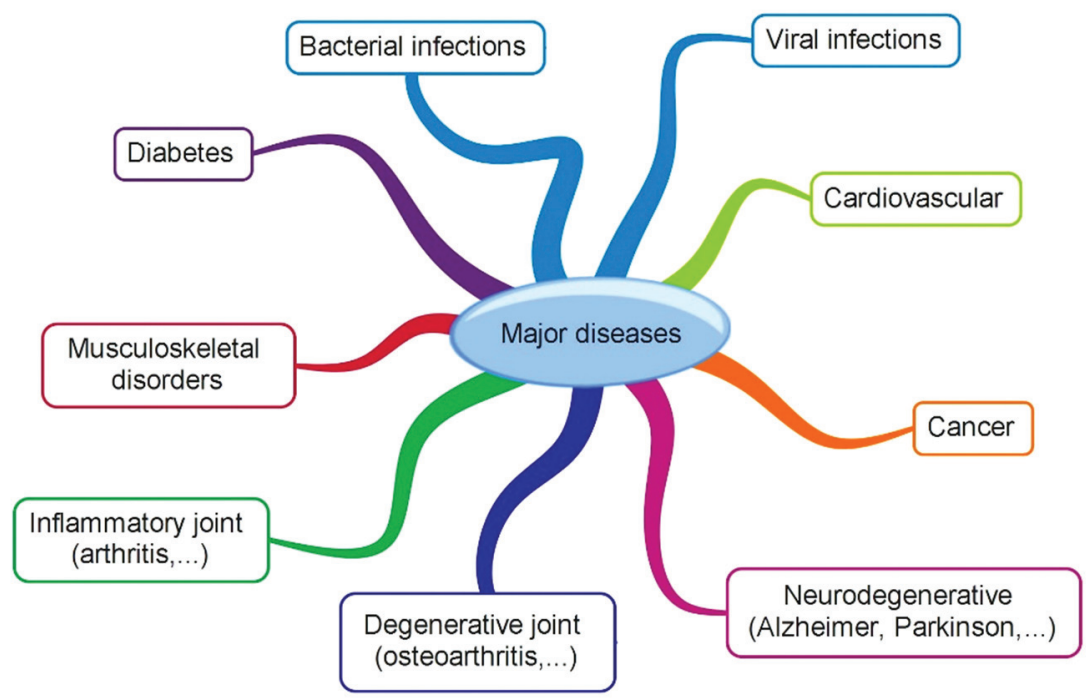

Figure 68. Major diseases touching a large part of the population.

\section{Diagnosis}

Diagnosis is the first step in identifying a disease or a health disorder. Diagnostic techniques should be specific, reliable, fast and accurate. The risks of "false positives" or "false negatives" at the borderline of the detection threshold should be minimized as much as possible.

Diagnostics include both in vitro techniques and in vivo techniques. Medical imaging is an example of an in vivo technique because it is performed directly on the patient. A blood analysis is an example of an in vitro technique since it is done on a sample taken from the patient. An important issue is to have non-invasive or minimally invasive techniques for both types of techniques.

\section{Imaging}

Imaging techniques which allow the investigation of a patient anatomy and, in some cases metabolic processes, are exceptionally powerful diagnostic tools (Figure 69). Functional imaging provides the ability to watch an organ function in real time. It allows the study the kinetics of biochemical and physiological phenomena. More recently molecular imaging allows visualizing the cellular function, genes and proteins directly or indirectly. They provide an unparalleled ability to identify dysfunctions of parts of the 

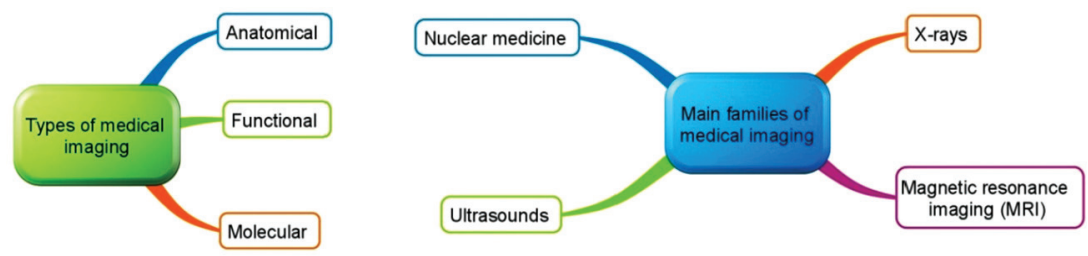

Figure 69. Types of applications of medical imaging (left hand side) and main families of medical imaging techniques (right hand part).

body and to anticipate appearance of disease. Modern Imaging techniques are highly accurate thanks to improvements in detection methods and computerized image treatment. The latter also allows most images to be digitized, leading to better storage and access to the data. While attempts are made to be as non-invasive as possible, these techniques sometimes require tracers to be injected into the patient.

The families of medical imaging which are mostly used are also indicated in figure 69 .

The use of X-rays is an old technique, but has improved greatly in our era. The aim is to decrease as much as possible the irradiation dose administrated to the patient and get the best spatial resolution.

Magnetic resonance imaging is a field in strong development with the advantage of being painless to the patient. Ultrasound techniques are increasingly being in many pathologies or to follow pregnant women. Nuclear medicine imaging techniques encompasses techniques based on radioisotope decay. The most common are scintigraphy, requiring injection or ingestion of a radioisotope, SPECT (Single-photon emission computed tomography) or PET (positron emission tomography).

It is often necessary to use several imaging techniques to make a diagnosis because they reveal different or complementary aspects of the health disorder.

The introduction of nanotechnology into imaging techniques through the development of new microprocessors, detectors and electronics is a natural evolution of imaging techniques. However, at the same time nanotechnology is playing a key role in providing new methods to enhance diagnostic imaging. The use of nanoparticles (which have large surface areas relative to their volumes) to deliver imaging agents to the desired site in the body, provides the ability to achieve a great density of interaction sites for interaction with molecular agents or particles. This can result in stronger imaging signals that can be detected outside the body of the patient. Dedicated nanoparticle structures with attached proteins or other molecules can detect indicators of disease at an early stage. It is also possible 
to create multifunctional products which can be used in different imaging techniques. Functionalized nanoparticles can have both a diagnostic and therapeutic potential. This property is often referred as theranostic.

A few examples of the use of nanoparticles in imaging:

- Gold nanoparticles are used in research to detect colorectal cancer, especially those which are difficult to see on colonoscopy. The detection is based on Raman spectroscopy. They are particularly useful because their surfaces resist oxidation and can be functionalized with different molecules.

- Iron oxide nanoparticles coated with dextran, a complex polysaccharide made of many glucose molecules, are used to target lymph nodes in diagnostic magnetic resonance imaging (MRI) and to detect cancer spread. These nanoparticles are taken up in the lymph nodes by normal cells but not by cancer cells. A MRI of the patient is performed before injection of nanoparticles and a second one is done 24 hours later. Lymph nodes that accept the nanoparticles become black while cancer cells stay bright.

- A new tumor detection technique, photo-acoustic imaging employs carbon nanotubes or gold nanoparticles which have been modified to emit sounds when appropriately stimulated by laser light. This light is used to heat the particles which produce an ultrasound signal that can be detected outside the body of the patient. The temperature reached is kept moderate in order not to hurt the tissue.

Structures in which gold nanoparticles are deposited electrostatically onto the surface of a PLA (polylactic acid ${ }^{9}$ ) microcapsule allow ultrasound contrast imaging. This structure has the potential to operate as a theranostic agent as well because it is also a photo hyperthermia agent allowing photothermal therapy in cancer treatment.

\section{In-Vitro Diagostics}

Presently diagnostic work typically has to be done in specialized laboratories and obtaining results can require hours or days. Required sample sizes can be large. Nanotechnology has an important role to play in the whole diagnostic process. The use of miniaturized laboratories, such as lab-on-chip systems which are in development and based on microelectronic techniques, can quickly provide a result using a very small amount of sample. Using smaller samples of body fluids or tissues makes the analysis 


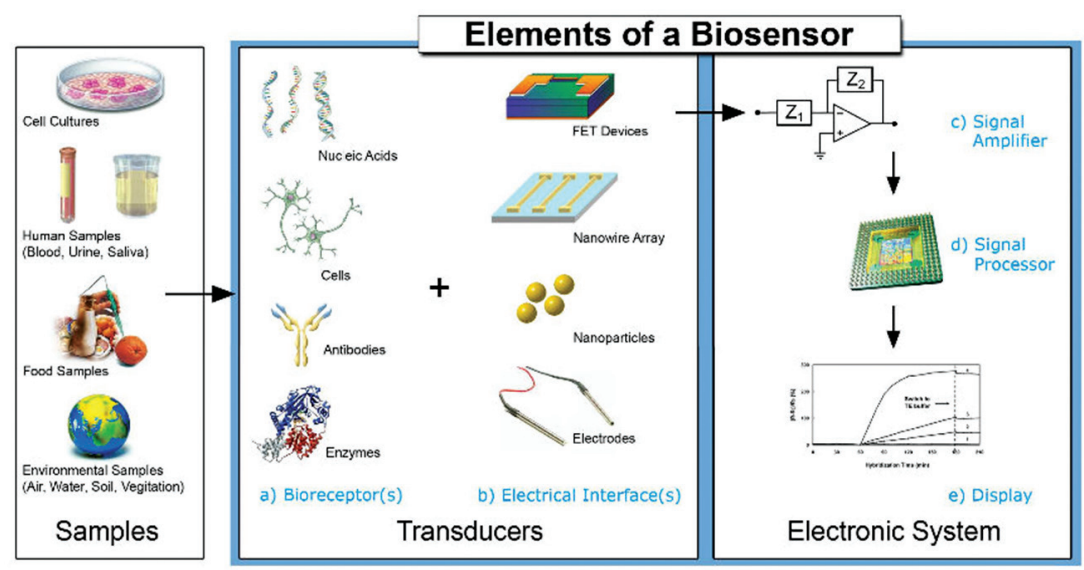

Figure 70. Biosensor system and components. Image from Wikimedia Commons (http://commons. wikimedia.org). Author Dorothee Grieshaber.

less invasive for the patient. The analysis can be done in a serial manner as it is done today, but also in parallel manner using arrays of miniaturized detectors when possible. Miniaturized analysis systems, including nanoscale components, will provide faster, cheaper results while producing less waste. Such low cost high speed analysis systems will soon become routinely available. The final goal is to make these capabilities directly available at the doctor's office.

\section{Biosensors}

Sensors are analytical devices which are able to recognize a specific chemical or biological species present in a sample, for example in a small quantity of blood from a patient. When the sensing is based on biomolecular recognition, the sensor is called a biosensor. There are several different types of biosensors based on different techniques of recognition such as antibody/antigen, enzyme based, hybridization with a strand of DNA, etc. The main elements of a biosensor are displayed in figure 70. The transducer transforms the signal resulting from the interaction between the analyte and the biological element into a signal which can be easily measured. This signal is amplified, processed and visualized by an electronic system.

A simple example of biosensor based on a cantilever is shown in figure 71. The free molecules are supposed to bind specifically to the molecules attached on 

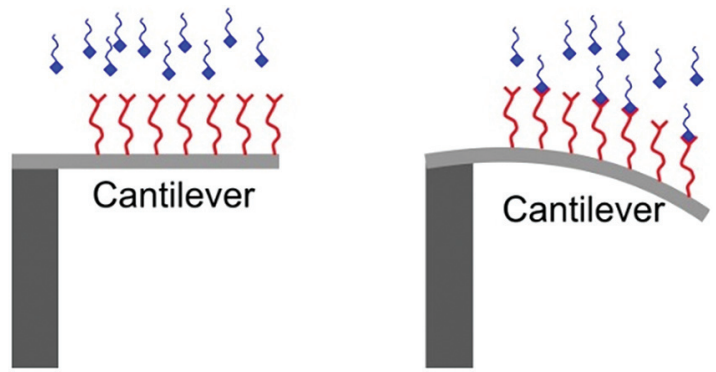

Figure 71. Principle of cantilever detection. The free molecules are supposed to bind to the molecules attached on the cantilever. When this happens the weight on the cantilever increases and it bends. The bending can be measured, by an optical method for example.

the cantilever. When this happens, the cantilever bends and information can be obtained from the measurement of the bending of the cantilever.

Nanoparticles of various types; gold or silica nanoparticles, quantum dots, fullerenes, etc. can be used in biosensors. Biological molecular species acting as sensors are attached to the surface of the nanoparticles. By the usual biological lock-and-key mechanism, they are able to detect the molecules of interest. The signal generated by the recognition mechanism can vary: a change of color, a change of mass, a change of emission properties etc.

Carbon nanotubes and nanowires can also be used in biosensors to detect a wide range of biological and chemical species. Viruses or proteins can be detected using these sensors which are often based on a change of electrical conductivity and employ semiconducting materials.

Although most of the biosensors developed from nanoscale materials are still in the laboratory, it is expected that they will enter operation at the patient scale during the next decade.

Microarrays of biosensors can be manufactured using the techniques of microelectronics. They can be used as diagnostic devices for DNA analysis, protein detection, virus detection, etc. Microarrays can consist of hundreds of biosensors acting independently and allowing specific detection of different bio-analytes within a mixture. Such microarrays can be used as screening tools, a function especially useful in new drug development.

Figure 72 introduces three families of microsystems containing nanocomponents which are under development. 


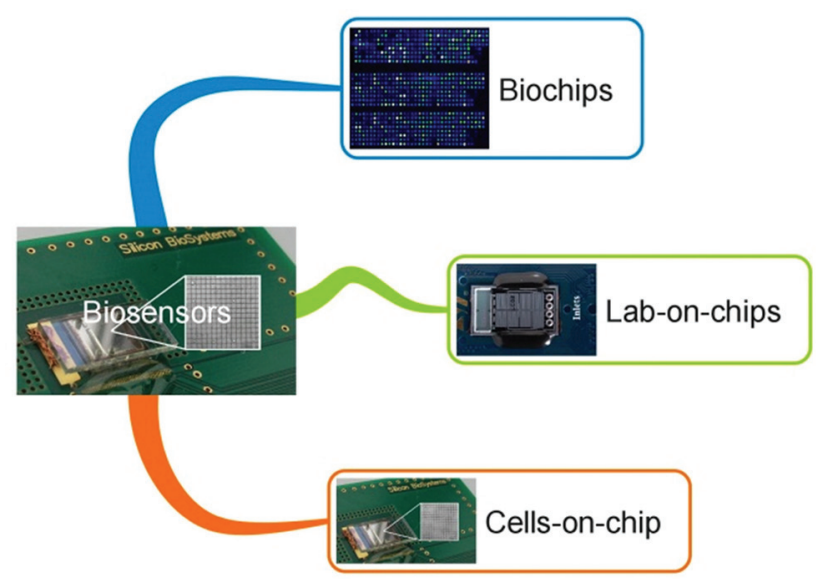

Figure 72. Different families of microsystems based on micro and nanoelectronics techniques and dedicated to diagnostics. The small images shown are courtesy of CEA/LETI (France).

\section{Biochips}

Genetic biochips (DNA-chips) are microarrays of sensors with the ability to perform a large number of analyses at the same time. Each sensor consists of a short strand of DNA. DNA-chips are able to quickly decode genes. When they are put into a solution to be analyzed, the analytes hybridize with the strands of DNA fixed on the microarray. A DNA-chip consists of thousands different nucleotide sequences arranged in a two-dimensional matrix. Biochips have been used to identify the genes of human DNA or of other living species. They can be used to detect the genes expressed by a cell or a tissue giving the ability to detect breast or prostate cancers, for example. They can also be used to detect proteins and in toxicological studies.

In some cases, the concentration of DNA in a sample is not always large enough to be detected safely or perhaps to be detected at all. There does exist a powerful technique, the Polymerase Chain Reaction technique, PCR, which can actually multiply the initial pieces of DNA to be detected: A single strand or a few strands of DNA can be multiplied by several orders of magnitude using PCR. In this in vitro technique thousands to millions of copies of a DNA sequence can be obtained. This amplification makes the DNA visible to the analysis system. 


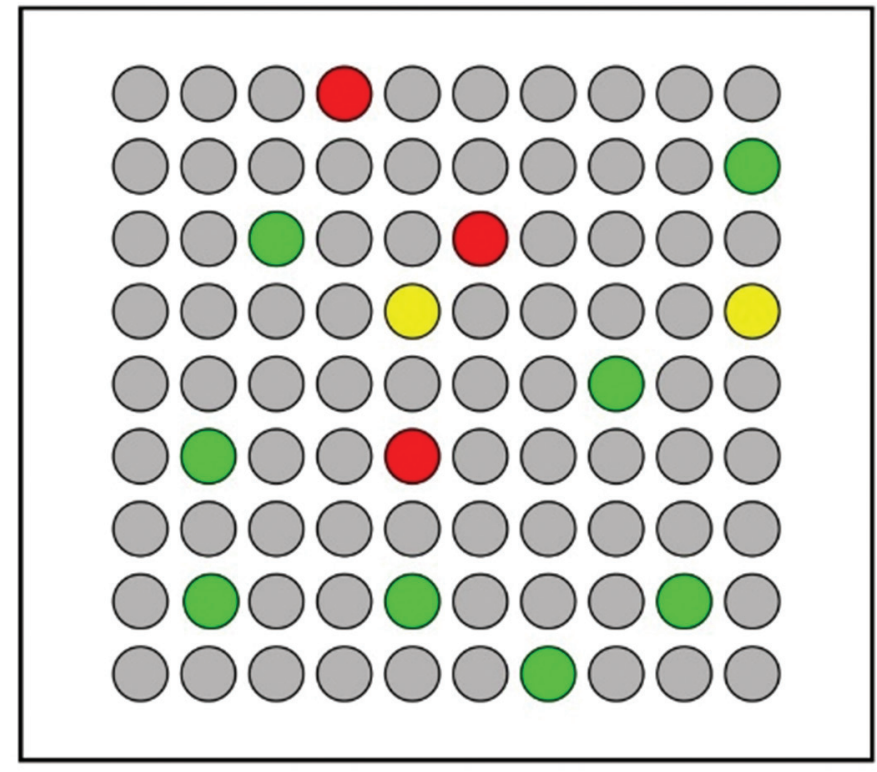

\section{No Hybridization}

\section{Hybridization from normal tissue}

\section{Hybridization from cancerous tissue}

\section{Hybridization from normal and cancerous tissue}

Figure 73. Schematic illustration of a DNA-chip.

PCR is performed in a vessel containing the DNA target to be amplified, a large concentration of olinucleotides (called primers) specific to the sequence investigated, the DNA polymerase and a mixture of the 4 bases which are the DNA building blocks. The PCR proceeds through a sequence of cycles, each of them comprises 3 steps lasting a few minutes: The cycle is repeated about 40 times, each time doubling the amount of DNA. About 100 billion copies can be obtained after 40 cycles. This process can be completely automated. Unlike some previous techniques it does not require the presence of bacteria.

Biochips such as these are used to detect oral cancers, for example. An oral cancer is usually discovered by dentists and oral surgeons and requires a biopsy of the cheek. The sample is sent to a laboratory and the result is received several days later. A nano-biochip recently developed in the US permits a painless test and the result is readily obtained in a quarter of an hour. Early detection of malignant or premalignant lesions is important 
because early treatment leads to a larger probability of survival. Early detection accords a 5 -year survival of $90 \%$. This figure drops down to $60 \%$ if the lesions are detected later.

Figure 73 explains schematically the way a DNA-chip is used in a small array. There are different spots indicating the probes hybridized on the array. Colored spots indicate that the probes have been hybridized. In this illustration it is supposed that red and green correspond to genes of the cancerous and normal tissue, respectively. Yellow spots correspond to a hybridization of both cancerous and normal tissue. This is because there are many probes located at a node of the array and not a single strand of DNA.

\section{Labs-on-chips}

Labs-on-chips, also called micro-total-analysis systems, integrate several chemical and biological analytical functions on the same chip. These devices are made of micro and nanocomponents manufactured with micro-electronic techniques. Labs-on-chip manipulate tiny volumes of biological fluids to perform analyses similar to those made in conventional laboratories. Miniaturized sensing systems and fluid control are essential to these devices.

Labs-on-chips are not simply miniaturized versions of conventional macroscopic laboratory techniques. They actually exploit the possibilities of new physical phenomena which appear at small length scales. Since the volumes are very small, for example in the nanoliter or femtoliter range, liquids behavior is different from that exhibited on the macroscopic scale as discussed in chapter 3. For example, since fluid dynamics is dominated by laminar flow, it is possible to create concentration gradients in nanoscale devices which are not achievable at the macroscopic scale. A good understanding of microfluidics and nanofluidics is essential to the development of devices performing similar analyses to those made in conventional laboratories, but much faster and at a substantially lower cost. Small samples and less reagent volume are required to perform the analysis. This produces less waste and, in the case where the sample is taken from a patient, is less intrusive. Since the volume analyzed is small and compact, the analysis is fast because the diffusion distances are much smaller than in macroscopic samples. As with biochips, it is possible to parallelize the measurements and build an array of labs-on-chips to get a high output rate. A mass production of labs-on-chips is possible using micro-electronic techniques. This lowers the cost substantially and could provide disposable chips. 


\section{Design considerations}

\section{Advantages}

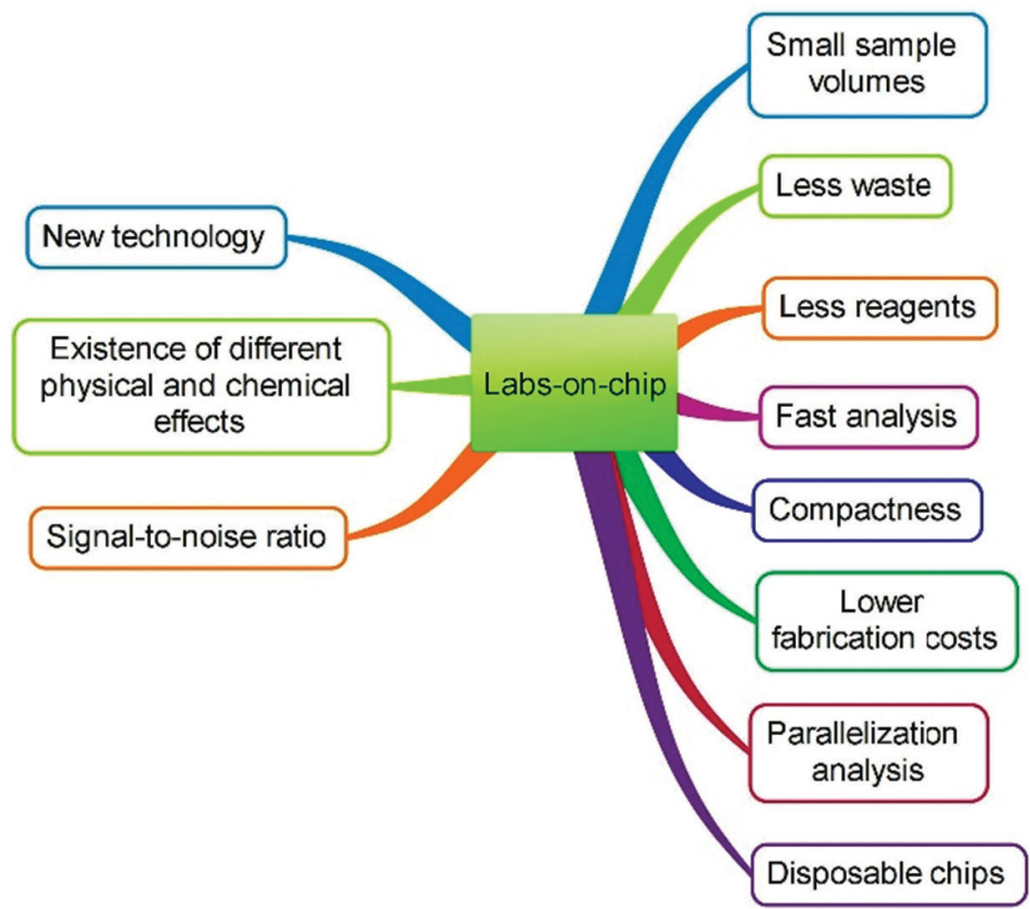

Figure 74. Design considerations and advantages of labs-on-chips compared to conventional technologies.

The first lab-on-chip system was a gas chromatographic microsystem developed in 1975. Developments in this field greatly accelerated about two decades ago. The main commercial applications up to now are in the medical and biological fields. However, applications in several other fields, environment, defense, chemical synthesis etc. are envisaged. ...

There are a lot of advantages to be realized with the development of labs-on-chip devices. Some of them are indicated in figure 74. Also indicated are some of the considerations to be addressed in such research. This is a new technology still in the state of infancy. The appearance of new physical and chemical processes at the nanoscale (see chapter 3 ) necessitates different designs then those existing in conventional laboratories. Furthermore, scaling down detection systems can reduce the signal-to-noise ratio requiring additional development to retain adequate sensitivity. 


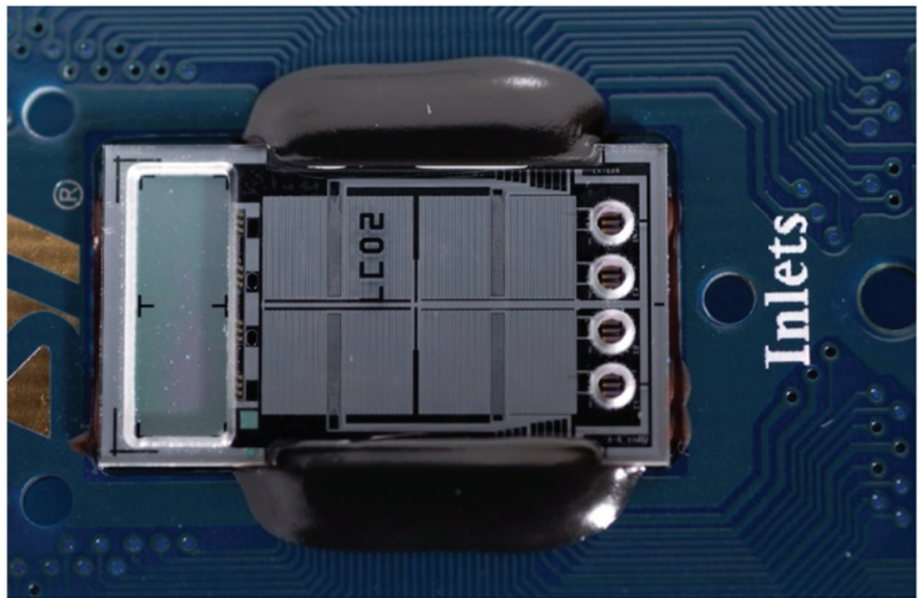

Figure 75. Lab-on-chip dedicated to genetic diagnosis developed by CEA and ST-Microelectronics. Image courtesy of CEA/LETI (France).

An example of lab-on-chip developed by the CEA and ST-Microelectronics is shown in figure 75 . This device is dedicated to genetic analysis. On a single chip it integrates sample preparation using the PCR technique and analysis using hybridization of oligonucleotide probes.

\section{Cells-on-chips}

Cells-on-chips couple living organisms such as cells with semi-conductor technology. Due to receptors on their surfaces cells are able to respond to changes in their environment with high selectivity and sensitivity. In cellson-chips, a change in the cell metabolism or morphology is transduced and analyzed using micro- and nanoelectronics. The cells are the sensing elements and can be used for pathogen detection and toxicology screening, as well as in research to better understand how cells function. Cells-on-chips are organized in arrays, allowing parallel analyses and high speed screening. The key point in cell-on-chip development is to interface living material with inert material. It is for example possible to connect neurons with electronic transistors or to grow cells in microdroplets to create an array of living sensors. These are usually $2 \mathrm{D}$ arrays.

Drug development is an important issue in the health domain. Drugs have to be effective against targeted health disorders but must have minimal negative side effects. Normally a first screening of drug candidates is 
done to find the best candidates. This is done in in vitro experiments. Conventional in vitro platforms try to identify the different signal molecules which are involved in the interaction between a drug and cells but rarely can mimic the cell-to-cell interactions in the body or the extracellular environment. There is now an effort to develop new research platforms based on more complex sets of cells to mimic the $3 \mathrm{D}$ scaffolding of cells to form tissues or organs. Organs-on-chips systems are being developed to mimic the function of livers, hearts, blood vessels, lungs, kidneys, muscles, etc. The liver is particularly interesting for studies of drug metabolism and toxicity. One of the advantages of these developments is to reduce the necessity for animal experimentation.

\section{Summary}

Treating a health problem begins with timely and accurate diagnosis. The less invasive the diagnosis technique, the more palatable it will be for the patient. Therefore, non-invasive imaging and small volume sampling are techniques which have many advantages. Presently in-vitro diagnostic work typically has to be done in specialized laboratories and obtaining results can require hours or days. Required sample sizes can be large. Nanotechnology has an important role to play in the whole diagnostic process. Micro- and nanotechnology allow a natural evolution of conventional diagnosis techniques and can provide rapid and reliable results economically. They also provide exciting new capabilities for research and development of new treatment modalities. Using miniaturized laboratories, such as lab-on-chip systems which are in development and based on microelectronics techniques, can quickly provide a result using a very small amount of sample. Using smaller samples of body fluids or tissues makes the analysis less invasive for the patient. The analysis can be done in a serial manner as it is done today, but also in parallel manner using arrays of miniaturized detectors when possible. Miniaturized analysis systems, including nanoscale components, will provide faster, cheaper results while producing less waste. The final goal is to make them available at the doctor's office. Such low cost high speed analysis systems will soon become routinely available. 